\title{
Thoughtful Drawings: A Computational Model of the Cognitive Nature of Children's Drawing
}

\author{
Ed Burton
}

\author{
The Centre for Electronic Arts, Middlesex University, Barnet, London EN4 8HT \\ E-mail: ed1@MDX.AC.UK
}

\begin{abstract}
An interpretation of children's drawing is presented that is based on active perception of the world and the construction of an equivalent self contained two-dimensional world. This model is implemented in a computer program called Rose (Representation Of Spatial Experience). Inspired by the drawings of young children, Rose simulates the perception of the general form and structure of three-dimensional computer models and constructs equivalent childlike two-dimensional representations from them. Rose serves to illustrate the plausibility of the constructive process as a model for real children's drawing and in addition serves as an alternative approach to traditional computer graphic rendering.
\end{abstract}

Keywords: children's drawing, experience, perception, representation, generalisation, feedback.

\section{Introduction}

Children's drawing by its very naïveté is most beautiful, endearing and instructive because the developing cognitive operations involved are relatively unadorned by cultural convention. A model of children's drawing is proposed that links perception and representation via an active process of construction. This is contrasted with the mechanical projective model of image making that has dominated western post Renaissance art and traditional computer graphic rendering.

The proposed model of children's drawings is implemented in a computer application. This project arises from a love of children's art and a desire to capture some of its beauty. The development of a working model furthers understanding of the theoretical model. The working model serves both as an illustration of the theory and an experimental tool that can suggest further development of the model.

The following sections discuss why children's drawing is of interest and give an interpretation of the drawing process which results in the 'constructive' model. A computer implementation of the proposed model is presented with observation, analysis and evaluation of the resulting behaviour.

\section{Children's Drawing: A Constructive Approach}

\subsection{Why Study Children's Drawing?}

As soon as a child is old enough to grasp a crayon in its fist it is compelled to make a mark. Most children draw prolifically and creatively from this moment on. Children's drawings are irradiated with beauty, humour 
and poetry.

In addition the drawings are interesting, not only because they remind us of our own lost innocence, but as symptoms of the more general cognitive phenomena of life. The elementary forms employed are easily distinguished from the complex objects they depict. It is immediately apparent that there are processes far removed from the mechanical projection of the camera. Children's naïve and relatively untutored nature helps to disclose the basic mental processes by which we understand the world about us. Paul Klee recognised the value of children's drawings and in 1912 said of them:

"The more awkward they are, the more instructive an example they offer us." [1]

\subsection{The Constructive Model}

For the purposes of this paper a new term is introduced to describe the drawing process: Constructive representation. This owes its greatest debt to Arnheim [2,3,4] whose explanation of Gestalt psychology was "sufficiently complex to apply to both perception and representation" [5]. The term Constructive is chosen primarily to contrast and sequester the prevailing projective interpretation of images. Klee eloquently summarises the philosophy behind the theory:

"Art does not reproduce the visible but makes visible." [1]

In essence, the representation must construct an equivalent world within the graphic medium from a vocabulary of forms that are translations of the artist's perception of the real or imagined world.

Central to the constructive process is the model of perception described by the Gestaltists who argue that perception consists of adding meaning to the unstructured stimulus of the senses by brain or mental organisation in a process of deterministic inference; as Crick describes:

"...seeing is a constructive process, meaning that the brain does not passively record the incoming visual information. It actively seeks to interpret it" [6]

This involves intelligently presenting only those qualities of an object salient to each situation. Thus the resulting percept is a generalisation of the detailed sense data.

The process of translation from the percept requires that a vocabulary be developed "within its medium and according to the conditions of the medium" [3]. This vocabulary varies throughout each child's development but in its early stages it typically places strong emphasis on topological equivalence between drawing and subject with little emphasis on Euclidean or projective equivalence. Thus Forms are differentiated by simple relationships such as proximity and separation, openness and closure with little regard for absolute shape or position.

As the graphic vocabulary is developed within its particular medium it adopts the properties of that medium. The predominant property of a drawing is that it is two-dimensional. Influenced by this environment, children's 'naïve' drawings can be usefully interpreted as two-dimensional equivalents of the three dimensions of space that we inhabit.

The child's drawing rather than being a projected view or window into three-dimensional space represents its own self contained two-dimensional world like the "Flatland" described in Abbott's mathematical fantasy novel [7]. Thus when a closed form is used to represent a solid volume, the perimeter line does not depict the projected horizon of that volume, instead it represents the entire surface of the volume. This model of children's drawing correlates with the conspicuous lack of occlusion and the frequent use of 'X-Ray' drawings such as those of people inside houses and food inside stomachs. 
A characteristic of the projective methodology is that sequence of production has little effect on the final picture. Conversely sequence is of paramount importance to the constructive model. With no global coordinate system it is difficult to anticipate isolated positions. For this reason there is an imperative to add new forms by accretion to existing forms [8]. The result is that a drawing grows outward from a core or series of cores. This sequential process of relating new forms only to previous forms also introduces the concept of feedback. Building on the consequences of previous marks leads not only to possible error and confusion but also to many creative discoveries of form.

\section{Computer Implementation of the Constructive Model: Rose}

Rose (Representation Of Spatial Experience) is a computer application that embodies many of the preceding ideas in a working model of the constructive process.

Rose is a metaphor for the human processes of perception and representation. As such, when terms such as experience, perception or awareness are applied to Rose they are being used in this metaphorical sense.

Rose's task is simply to perceive three-dimensional forms and construct two-dimensional representations from them in a similar way to that of a young child. Rose has been designed as an interactive application (figures $1 \& 2$ ). Thus the maximum scope for investigation and experimentation of Rose's characteristics is afforded. Rose has been developed on an Apple Macintosh Quadra 650 personal computer, the programing language used is Object Orientated C.

Rose is not intended to be a model of any part of a child's mind. Rose is a representation of personal ideas about just a few vital elements of the human experience of drawing. In essence the computer program becomes a medium for a self portrait, an attempt to understand the self.

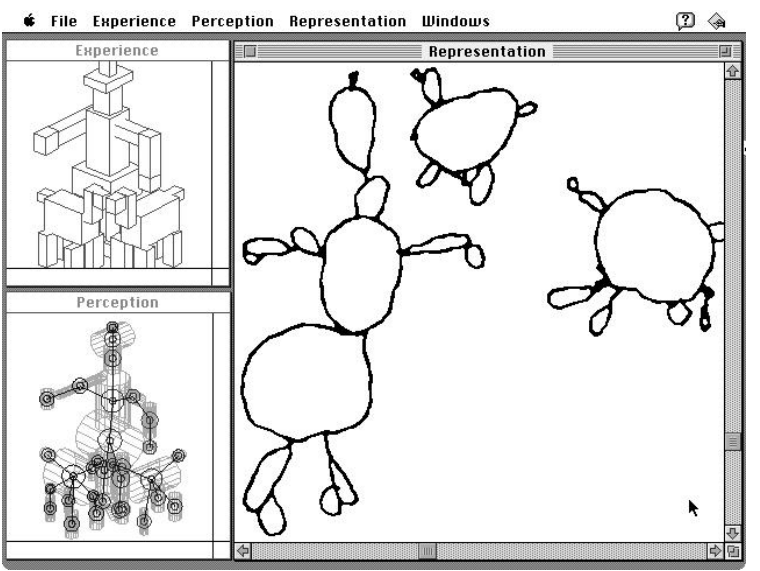

Figure 1. Rose's user interface.

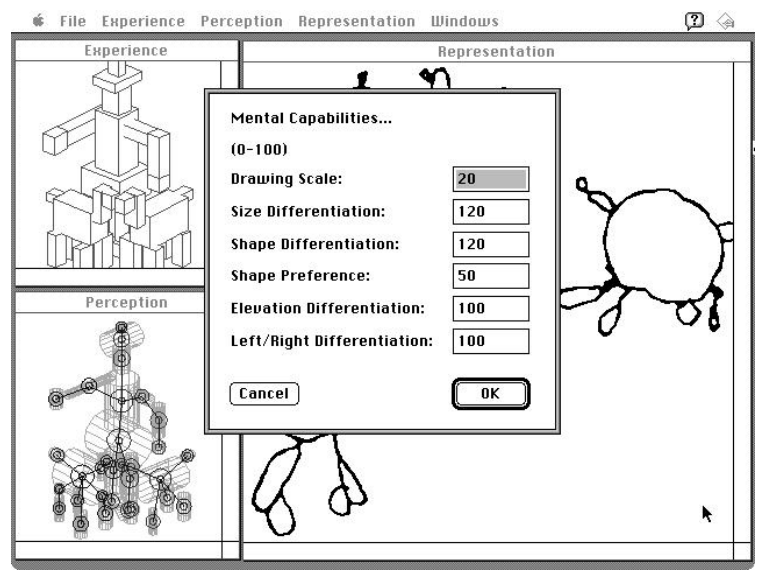

Figure 2. Modifying Rose's “mental” capabilities.

\subsection{Schematic Process: An analogy for Experience, Perception and Representation}

Rose uses primarily three different domains of spatial awareness (figure 3). Experience is Rose's equivalent to contact with the outside world. Perception is an interpretation of the Experience combining canonical shape and topological relationships of volumes. Representation is the drawing rendered from Rose's Perception. Each of these will be dealt with in turn. 


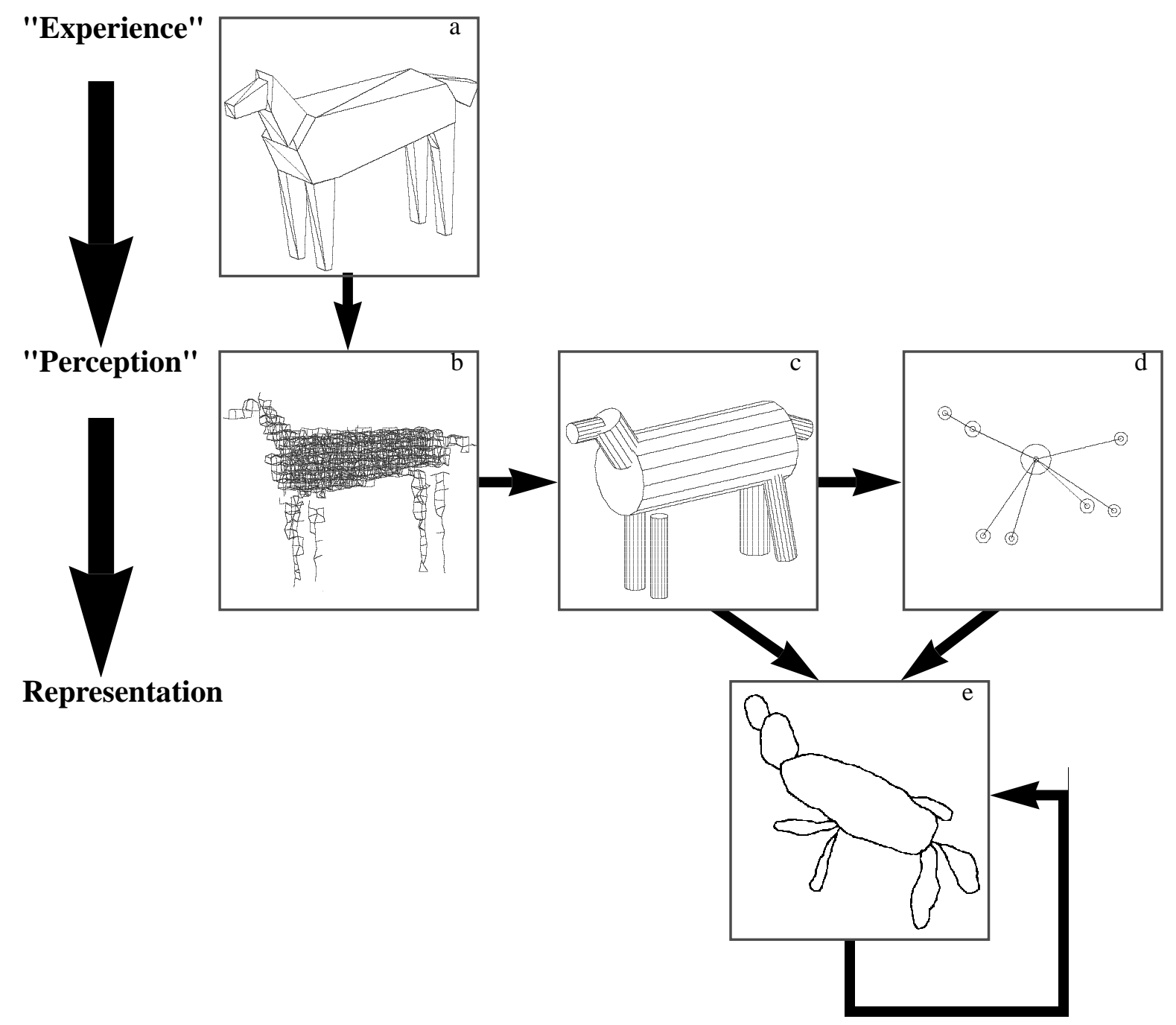

Figure 3. Schematic process.

\subsection{Experience}

Our experience of an environment is multi-sensory and accumulated over time. For this reason no single sense has been simulated. The senses most pertinent to representation are sight and touch. These experience only the surface properties of objects. Thus it is assumed that over time we can access the three-dimensional shape of all surfaces in an environment independently of a fixed view point. For this reason, Rose's 'sensory' input is in the form of three-dimensional boundary representation (B-Rep) models as DXF files (figure 3.a), converted by Rose into a convenient three-dimensional model database.

The experience model describes only the geometry of planar surfaces, with no explicit information about how these surfaces combine to create volumes. Note that this is how most traditional computer renderers represent three-dimensional form (with the exception of CSG modelling) [9] as they are only concerned with projecting the surface appearance of form. 


\subsection{Perception}

Based on the preceding research Rose's Perception of the Experience is based on three main premises. Firstly that the primary processes of perception are topological in nature, that is, based on simple relationships like proximity and separation. Secondly that perception generalises or abstracts the detailed data from the senses. Lastly that this results in the 3D Concept or Model which summarises an object's canonical form independently of any viewpoint. Rose's perceptual process typically takes about 10 seconds to achieve.

Rose's perceptual process consists of three consecutive levels of abstraction which will be dealt with in turn.

\subsubsection{Perceiving Nodal Networks}

During human perception even though experience consists only of information about surfaces, internal volumes and masses are perceived. Piaget among others has demonstrated that the primary processes of perception are topological [10]. For these reasons the first level of Rose's perception consists of transposing the experience model into a topological network of potentially many thousands of nodes. Each node represents a small region of space which has six neighbouring nodes in an orthogonal grid. Two neighbouring nodes are defined as proximate if the shortest line (link) between them does not intersect a surface, otherwise they are separate.

Continuous space is represented by proximity between all neighbouring nodes. The surfaces of the experience model separate pairs of nodes within this continuous space. This has the result of dividing the nodes into discrete connected networks of nodes describing an amorphous shape. Each of these networks corresponds to a bounded volume within the experience model (figure 3.b shows the links between all proximate nodes within bounded volumes).

All the nodes in a cuboid containing the model are stored as a one dimensional array that represents a threedimensional orthogonal grid. The links in the grid are represented by three bits in each node which record if it is proximate to or separate from its next neighbour along each positive axis direction. The program performs intersection tests only on the proximate links within the bounding box of each surface, making those links separate if they cross a surface. The individual volumes are then identified by a recursive search of each of the connected networks of nodes.

\subsubsection{Perceiving Canonical Shape}

Marr and Nishihara describe vision as "a process that produces from images of the external world a description that is useful to the viewer and not cluttered by irrelevant information." [11]. Rose's nodal network is a necessary interim stage for identifying the presence of volumes but it is very cluttered by irrelevant information, making it difficult to access the general salient features of volumes directly from their nodal networks.

Marr and Nishihara show that an object's surface does not have to be reproduced in order to facilitate recognition. They illustrate that objects can be adequately recognised when generalised as collections of cylinders. This has the advantage of discarding large amounts of particular information concerning surface properties.

Rose generalises each volume as being the single cylinder that fits its shape most closely, as can be seen in figure 3.c. Each cylinder is defined compactly by principal axis, extent and radius. The extent and radius will be used to judge the appropriate size and eccentricity of the form to be drawn. The elevation of the principal axis will affect the orientation of unattached forms.

For each volume the canonical cylinder is approximated by the cylinder with smallest radius able to enclose 
all of a volume's nodal network, passing through the network's centre of mass and chosen from a limited number of test orientations.

\subsubsection{Perceiving Volumetric Relationships}

Rose identifies the nodal networks corresponding to each volume by the analysis of many thousands of tests of proximity or separation on a "microscopic" scale. Now that Rose has identified each volume as a single cylinder, these individual volumes can be placed within a structure that records proximity and separation on a "macroscopic" scale.

Rose records a proximate relationship between any two volumes when their identifying nodal networks contain neighbouring nodes. This builds up a structural skeleton linking the volumes in a network (figure 3.d). This completes Rose's 3D Concept of an experience.

Notice that each level in the perceptual process contains less information specific to the detailed shape of the experience than its predecessor. Also Rose only performs many isolated "microscopic" tests of proximity and separation directly on the experience model. Nevertheless, by considering the many disparate tests globally or as a gestalt, Rose is able to deduce not only the general shape of volumes but also all subsequent topological properties such as continuity, order and enclosure on a "macroscopic" scale between volumes. This progression from large amounts of "low level" data to a small amount of "high level" information is a property common to many models of natural perception processes [12] typified by Marr and Nishihara's work on the recognition of three-dimensional shapes [11].

\subsection{Representation}

Rose's method of creating drawings is based on the constructive model of representation in young children described in section 2.2. To recapitulate the constructive model, Rose will create an equivalent world within the two-dimensional medium, rather than a projection of the three-dimensional world. This is done by translating from the percepts to a vocabulary of graphic form. The drawing must be constructed in a particular sequence as it is modified by feedback.

The drawings are output as high resolution (typically 840x840) bit map images. Like the majority of children, Rose creates line drawings. Each perceived volume is represented in the drawing by a single form which is a continuous pen stroke usually approximating an elliptical shape. Rose employs a 'turtle-like' drawing method [13] in which the 'pen' is advanced in small steps of constant length and varying angle. The drawing in progress is stored as a series of linked lists of points, one for each form depicted. A reasonably complex drawing takes typically five seconds to be drawn. To reflect the physical nature of the graphic medium a cellular automata algorithm is applied to the bit map image to simulate the effect of a felt tip pen on absorbent paper.

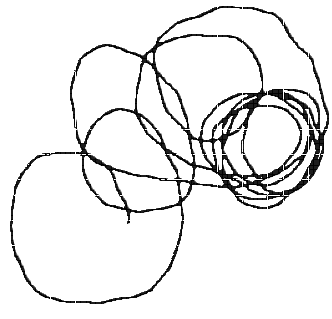

Figure 4.Scribbling

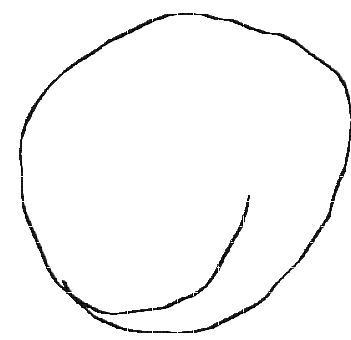

Figure 5. Closed form. 
Rose's program development consisted of two distinct phases: Evocation and Representation as follows.

\subsubsection{Rose's first phase. Evocation}

Rose's first phase consisted of developing a simple vocabulary of graphic form and developing an "awareness" of the picture to enable feedback to occur. The first marks made (figure 4) are the result of unrestrained "motor" activity which, as in very young children, produces scribbling. This is achieved by changing the pen direction at each step by a randomly perturbed value.

The scribbling is modulated by Rose's first level of feedback, detecting if a line has been crossed. Rose checks for intersections between the pen and all previous lines stored in the linked lists. If the the pen intersects the current form the form is finished. This creates a closed form (figure 5), used universally by children to represent undifferentiated shape. Repeated use of even this very simple vocabulary has created some surprisingly evocative petroglyph-like forms (figure 6).

Alternatively Rose can use the feedback from line crossings to avoid forms. Being able to differentiate between the line of the form being drawn and other forms makes it possible to combine closure and avoidance. The ability to navigate the pen relative to distant points enables Rose to create closed forms that are distorted by previous forms but always attempt to return to their starting point (figure 7).

Having developed the ability to create closed forms "ballistically" Rose must be able to differentiate between forms. Rose can differentiate sizes and also vary the eccentricity of a form to create differentiation between large and small circular, elliptical and linear forms (figure 8). This completes Rose's basic graphic vocabulary.

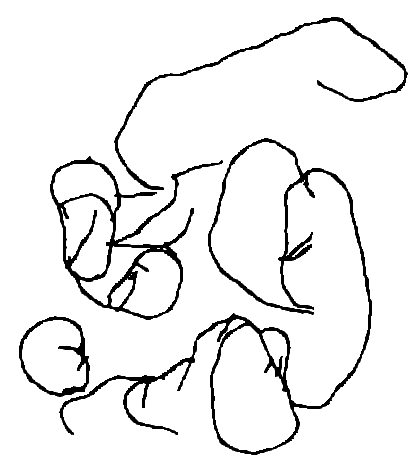

Figure 6. "Petroglyph-like" forms.
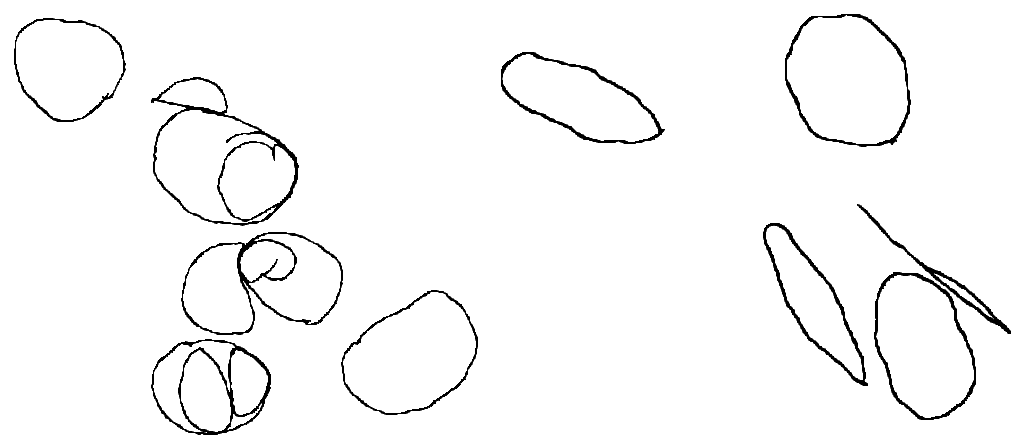

Rose creates a closed form by navigating the pen relative to the form's starting point. At the beginning of a form the pen should be heading directly away from its starting point. For a form to be closed it should end with the pen heading directly towards its start point. Constant transition of the pen's direction relative to the form's start between these two states along the desired length of the line would approximate a circle, Rose uses simple functional variations of the change in pen direction dependent on eccentricity (desired width/ desired length) to allow a continuous progression from approximated circle, through ellipse to straight line. 
Rose continuously samples the pen's distance from other forms and the edges of the page which it will try to avoid. The pen direction is also perturbed by a variable amount of Gaussian noise to simulate a child's poor motor control. This has the effect that a form can be considerably distorted from its "intended" shape. The fact that the navigation of the pen is relative to the form's starting point means that Rose always attempts to close a form even if it is distorted.

\subsubsection{Rose's second phase. Representation}

In the drawings Rose endeavours to construct, from the graphic vocabulary, equivalent structural relationships to those of the perceived 3D Concept.

To do this Rose must first choose an appropriate sequence to construct the drawing. van Sommers observes from many videotapes of children drawing that $98 \%$ start drawing from a common "core" of an object and work outwards by a process of accretion to existing forms [8], but gives no clear indication of what constitutes the "core".

Rose identifies an object as being a network of proximate volumes. The core of an object is taken as a volume with the maximum number of proximate relationships with other volumes, for example the torso in a human figure. This corresponds to Marr and Nishihara's choice of principal axis in a 3D Model consisting of many component axes [11, p. 280]. The remaining volumes of an object are sequenced by a recursive search growing outwards from the core. After observations by van Sommers, Rose has a secondary preference to draw from top to bottom.

Having chosen the sequence, Rose must identify the principal and secondary axis of each object. It is intended that Rose has a crude commonality with human experience, so the principal axis is always vertical to correspond with usual orientation under gravity. The principal axis is used to estimate the elevation at which a form should be attached to its neighbour.

The secondary axis is defined as the direction perpendicular to the principal axis (horizontal) in which an object's structural skeleton has maximum extent. It is used to test if connected forms are to be placed to the left or right of each other in the drawing. This procedure has the effect that Rose will usually draw a human figure with paired limbs placed symmetrically about the vertical torso, but draw a standing horse with paired limbs grouped at opposite ends of the horizontal torso. A projective interpretation would describe the human as drawn as seen from the front, and the horse as seen from the side. It is important to realise that for Rose this is not the case. Rose uses the secondary axis to identify the most informative way to differentiate between opposite sides of an object. The secondary axis does not define an external view point.

Having chosen the sequence and axes of each object Rose draws using the length and radius of the forms' corresponding volumetric model to plan the appropriate size and eccentricity of the ellipse to be approximated. If the form to be drawn has an active relationship (proximity or enclosure) with a form already drawn then it is accreted to that form according to its orientation about the objects axes.

As Rose, like a young child, does not use a global coordinate system [10] if a new form does not have a active relationship with a prior form it is placed randomly in any area of empty space but orientated according to the elevation of the principal axis of the forms' corresponding volumetric model.

Rose is prone to make confused drawings if objects are started too close to one another or the edge of the page. This lack of anticipation is evident in many children's drawings. 

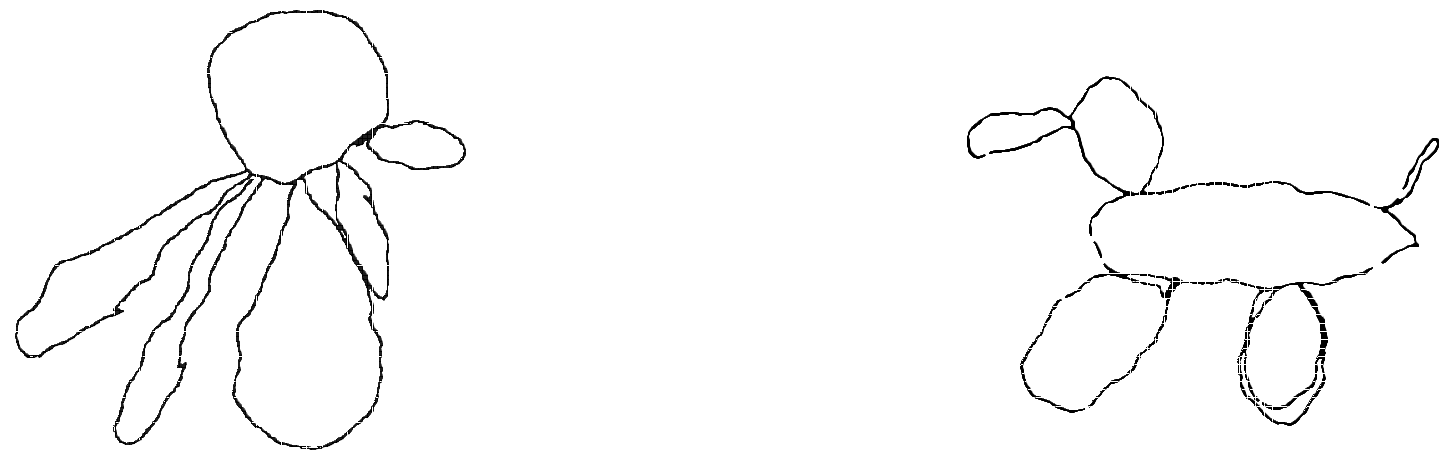

Figure 9. One of Rose's earliest attempts at drawing a human figure. Figure 10. An early drawing of a horse.

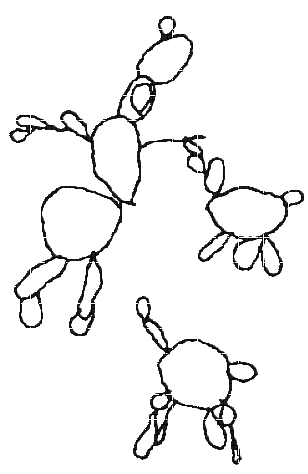

Figure 11. A woman with two dogs and a big hat.
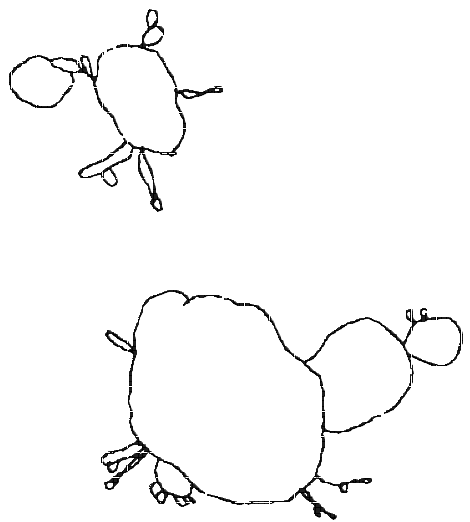

Figure 12. Farmer and cow.

\section{Behavioural Study of Rose}

Rose has been a prolific young artist. Above are some examples of Rose's drawing abilities (figures 9-12). The following drawings are compared with observations of real children's drawings.

Figure 13 is a drawing of a simple figure with hat and shoes drawn by Rose. Figure 14 is an original drawing by a child aged two years and eleven months. Notice that in both drawings limbs are drawn approximately perpendicular to torsos. Arnheim observes that child psychologists have erroneously identified the frequent gesture of such outstretched arms an indication of despair [3]. Rose, like the young child, will render limbs in this fashion regardless of their orientation in the experience model. This is a consequence of the constructive process as Rose certainly has no algorithm for emotional abandonment. At this stage the process of translation from percept generalises all possible orientations of limbs as being equivalent. Thus the angles are undifferentiated, being represented by the simplest angle offering the greatest contrast between elements, the right angle. Arnheim asserts that this is the process that operates in children's drawing.

Figure 15 is a conventional perspective rendering of the experience model of three animals. Figure 16 is Rose's interpretation of this model. The drawing illustrates Rose's ability to differentiate between size and shape in an attempt to derive the canonical shape of an object. The result is that the horse, giraffe and dog are easily distinguished from each other in the drawing. Notice also that there is no view of the experience model that does not obscure some parts. In the drawing however all parts are visible. Rose embodies the principle 


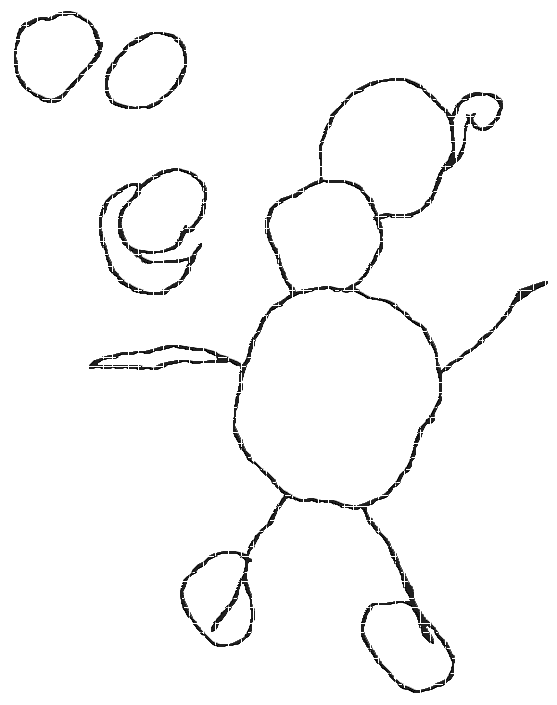

Figure 13. Figure with hat \& shoes.

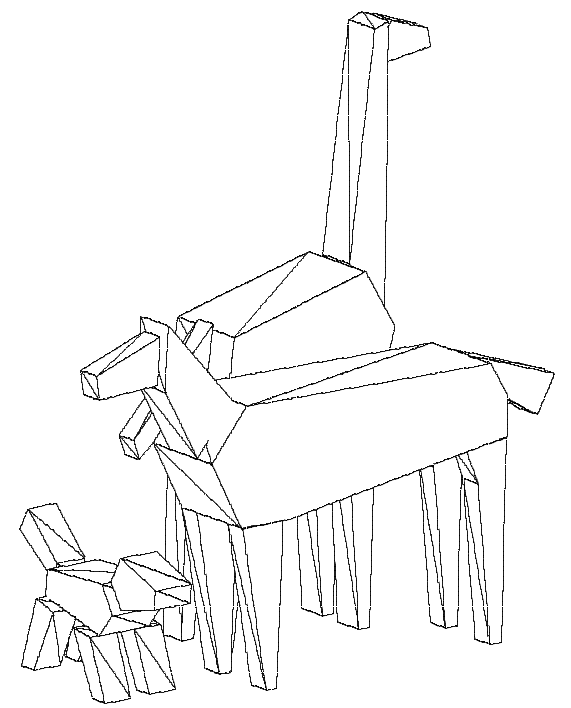

Figure 15. Experience of three animals.

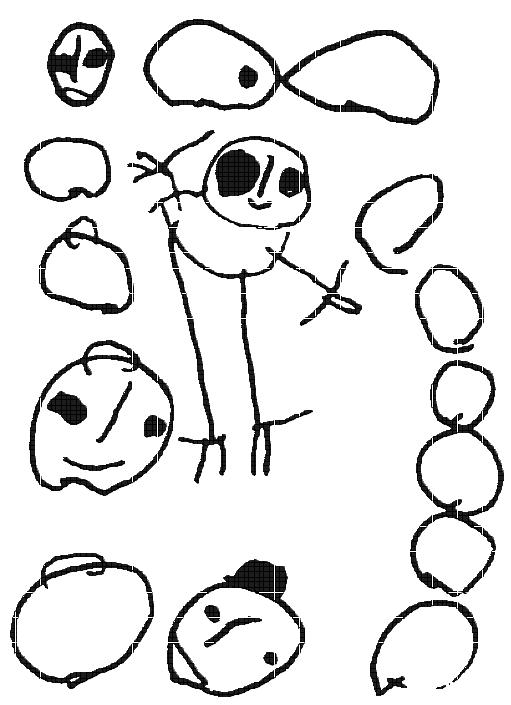

Figure 14. Forms, faces and figure.

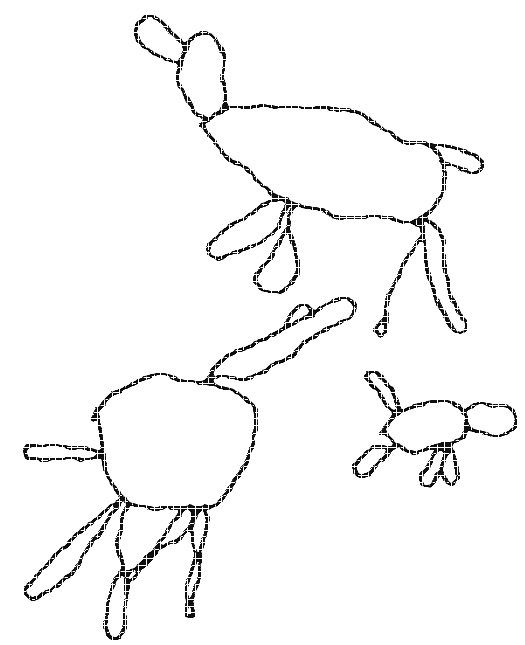

Figure 16. Drawing of three animals.

set forth by Klee and so apparent in children's drawing:

“...everything essential, even though hidden by optical perspective, appears on the plane." [1]

Rose's deviation from optical perspective is even more apparent when rendering the topological relationship of enclosure. Figures 17 \& 18 show Rose's experience and subsequent drawing of a person inside a house with "food" inside the body. The conventional rendering of the experience model reveals little. Rose's drawing is not an "X-Ray" or transparent view of the scene. Inspired by children's drawings, it is a constructed two-dimensional equivalent of the "imagined" scene. 


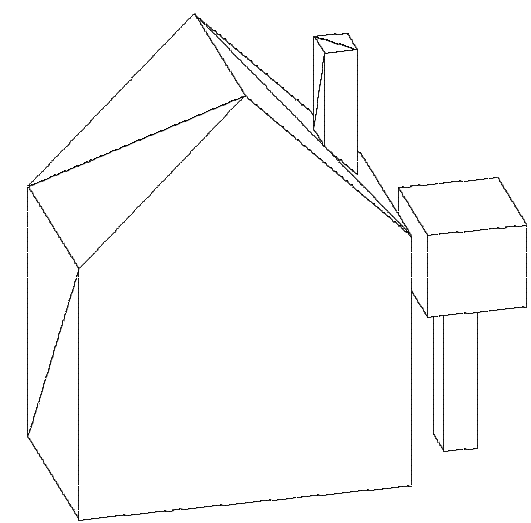

Figure 17. Experience of a house.

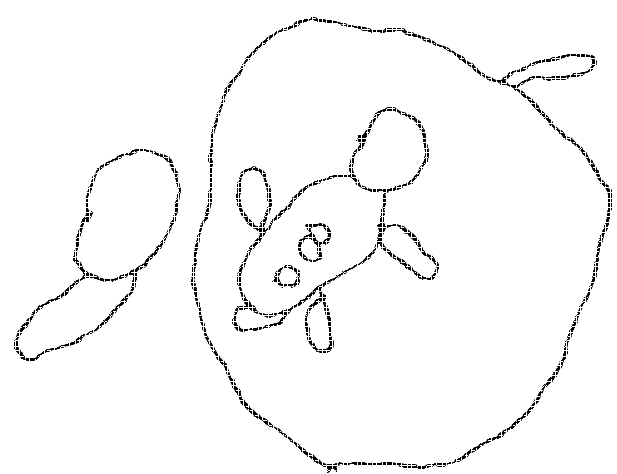

Figure 18. Drawing of a house.

\section{Evaluation of Rose}

\subsection{What has Rose achieved?}

Rose can "sense" the volumes implied by the surfaces of the experience model and find the principal axis, extent and radius that best approximate their shape. Rose uses the deduced information about these volumes and the relationships between them successfully to construct two-dimensional representations of them.

"Success" means that the drawings usually display a significant level of equivalence with the original experience.

Rose is a very limited model of children's drawing. Due to the central importance of representation, to simulate children's drawing completely would surely require a complete model of a child's mind (and body?). Obsessively literal about some characteristics but wholly ignorant of others, Rose lacks any scope for learning or spontaneous creativity. This very weakness is also one of Rose's strengths. One of the difficulties in studying children drawing is that they are so creative and learn so fast that it is all but impossible to isolate individual operations before the child invents a new approach.

Rose embodies an isolated set of rules that can be studied with repeatable observations. Even more useful as both an experimental and visual tool, many of Rose's rules can be modified through the user interface. For example it is possible to deplete Rose's ability to differentiate sizes. The result are figures with limbs as large as their bodies which look more childish.

At present Rose has two main weaknesses in the realm of perception and representation respectively. In perceiving volumes Rose is very literal in identifying perceptible parts. A perceptible volume must be completely bounded by surfaces. Thus if a large volume has an appendage, that appendage will only be perceived as a separate volume if it is separated from the large volume by a surface. Future development could include the ability to decompose complex shapes into perceptible parts.

Conspicuously lacking from Rose's representational skills is any sense of composition. Comparing figure 14 to figure 13 the real child's drawing contains an innate sense of compositional structure and balance. It would be desirable to develop in Rose more complex placement strategies to simulate what has been shown to be a universal predisposition to aesthetically balanced composition [14].

Despite its limitations, Rose has captured some of the strange innocence, verve and humour of children's 
drawings. This was the original ambition behind the project and it has been impossible not to feel some kind of paternal pride in witnessing Rose's difficult and faltering development.

\subsection{What can Rose teach us?}

Rose embodies a working alternative to the projective model of representation. Rose demonstrates that it is not necessary to have any concept of an external station point to produce recognisable child like drawings.

Rose also demonstrates that a representation can be constructed entirely from the distinction between the primary topological relationships of proximity and separation. Applied to a space it is possible to deduce from these two relationships all topological properties such as continuity, enclosure and order.

Rose attempts to isolate the salient features of an experience and represent just those generalised qualities. The drawings produced can prove more evocative than projective views of the experience because they are general enough to allow the viewer to invest meaning in them. They are also more directly communicative of the overall structure of an experience because they are uncluttered by the details of the experience model's three-dimensional surfaces.

In conclusion Rose as a young artist has created drawings that are lively, endearing and faltering like a child's. Rose as a model of representation implies that children's drawing is of an active, interpretive and constructive nature and that this results in drawings that are both evocative, communicative and thoughtful.

\section{References}

[1] Spiller, Jürg. Paul Klee Notebooks Volume 1 The thinking eye. Lund Humphries Publishers Limited. London. 1961.

[2] Arnheim, Rudolf.Visual Thinking. Faber and Faber Limited. London. 1970.

[3] Arnheim, Rudolf. Art and Visual Perception, a psychology of the creative eye. The New Version. University of California Press. Berkeley \& Los Angeles. 1974.

[4] Arnheim, Rudolf. New Essays on the Psychology of Art. University of California Press. London. 1986.

[5] Hagen, Margaret. The Perception of Pictures. Volume II. Dürer's Devices. Beyond the Projective Model of Pictures. Academic Press Inc. London. 1980.

[6] Crick, Francis. The Astonishing Hypothesis. The Scientific Search for the Soul. Simon \& Schuster Ltd. London. 1994.

[7] Abbot, Edwin. Flatland. A romance of many dimensions by A. Square. (2nd ed published 1884). Basil Blackwell. Oxford. 1978.

[8] van Sommers, Peter. Drawing and Cognition. Descriptive and Experimental Studies of Graphic Production Processes. Cambridge University Press. Cambridge. 1984.

[9] Mäntylä, Martti. An Introduction to Solid Modeling. Computer Science Press.Rockville. Maryland. 1988. [10] Piaget, Jean \& Inhelder, Bärbel. The Child's Conception of Space. Routledge \& Kegan Paul. London. 1971.

[11] Marr, D \& Nishihara, H. Representation and Recognition of the Spatial Organisation of Three-dimensional Shapes. Proceedings of the Royal Society of London. Vol. B. 200. pp. 269-294. 1978.

[12] Boden, Margaret. Computer models of mind. Computational approaches in theoretical psychology. Cambridge University Press. Cambridge. 1988.

[13] Abelson, H \& diSessa, A. Turtle Geometry. MIT Press. Cambridge. Massachusetts. 1982.

[14] Kellogg, Rhoda. Analysing Children's Art. National Press Books. California. 1970. 